\title{
STUDY OF ENERGY EFFICIENT SENSOR NETWORK USING AD- HOC COGNITIVE RADIOS
}

\author{
Abhinav Lall ${ }^{1}$, O. P. Singh ${ }^{2}$, G. R. Mishra ${ }^{3}$, Ashish Dixit ${ }^{4}$ \\ ${ }^{1}$ Department of Electronics and Communication Engineering, ASET, Amity University Lucknow Campus \\ ${ }^{2}$ Department of Electronics and Communication Engineering, ASET, Amity University Lucknow Campus \\ ${ }^{3}$ Department of Electronics and Communication Engineering, ASET, Amity University Lucknow Campus \\ ${ }^{4}$ Department of Electronics and Communication Engineering, ASET, Amity University Lucknow Campus
}

\begin{abstract}
An Energy Efficient network is proposed that consists of Ad-Hoc (mobile) Cognitive Radios (CR's) and infrastructure wireless sensor nodes. Sensor nodes within communication range of each CR are grouped into a cluster and the clusters of cognitive radios are regularly updated according to the random mobility of the cognitive radios. We reduce the energy consumption and end-to-end delay of the network by dividing by each cluster in disjoint subsets with overlapped sensing coverage of primary user (PU) activity. Respective subsets of CR provide target detection and false alarm probability. Substantial energy efficient is achieved by activating only one subset of the cluster, while putting rest of the subsets in the clusters into sleep mode. Additional gain in energy efficient is achieved by two promising propositions: Selecting nodes from the active subsets to sleep mode by scheduling. The sensor nodes for Real Time Spectrum sensing are chosen considering their respective time durations for sensing. We compare the proposed CR networks with existing approaches to demonstrate the network performance in terms of the energy consumption and the end-to-end delay.
\end{abstract}

Keywords-Cognitive Radios, Ad-Hoc CR Network, Sensor Network

\section{INTRODUCTION}

As per the Federal Communications Commission (FCC), utilization of the statically assigned spectrum varies from $15 \%$ to $85 \%$, depending upon spatio-temporal variations. In order for a secondary user, which cannot be active when the primary user (PU) is active, to utilize the spectrum licensed to a PU, the activity of the PU should be closely monitored. One possible approach is to use cognitive radio (CR) transceivers for spectrum sensing and sending their observations to a fusion center to determine the presence of the PU signal. However, this approach incurs high cost and high energy consumption.

A more appealing approach is to perform sensing via costeffective and dedicated sensor network. Use of the sensor network for spectrum sensing is being explored by regulatory bodies like the $\mathrm{FCC}$, which has invited experts to draft proposals for the use of a sensor network with low cost/energy/delay for enhanced spectrum sensing. Energyefficient spectrum sensing by a sensor network offers advantages such as more effective detection of a weak PU signal (by location diversity of the sensor nodes) and better protection of the PU due to high reliability in detection. Furthermore, this approach is more appropriate for mobile CRs where cooperative spectrum sensing is more difficult in the absence of a fusion center and cooperation between the CR users cannot be easily achieved. However, there are still certain challenges/disadvantages in such a network, which are yet to be resolved; examples are ownership of the sensor network, information dissemination by the sensor network, usage fees, etc.
The sensor network required for spectrum sensing should be a low-cost network consisting of a large number of spatially distributed sensor nodes equipped with sensing, processing, and communications capabilities. Because the sensor nodes are characterized by their limited resources (e.g., storage capacity and processing power, and typically nonreplaceable, limited-capacity batteries), efficient consumption of the energy, which affects network lifetime, is a major concern. The sensor nodes carry out spectrum sensing by means of energy detection, and report the results to the CR acting as a fusion center. The decision fusion at the CR employs the OR-rule, which decides the presence of the PU signal when at least one of the sensor nodes reports its presence. The effect of location diversity is more profound with more sensor nodes involved in spectrum sensing.

In this paper, a $\mathrm{CR}$ network (CRN) with disjoint subsets for each cluster of sensor nodes is studied as a solution to the problem - effective sensing achieved with high energy efficiency. The CRN is composed of ad hoc CRs, assigning mobility to $\mathrm{CRs}$ to be more general, and infrastructure sensor nodes. An ad hoc CR, which is a cluster head, is surrounded by a cluster of infrastructure sensor nodes within one-hop communication range of the $\mathrm{CR}$, and each cluster is further partitioned into subsets. To achieve energy efficiency, sleep-wake scheduling for the subsets based on the statistical behavior of the PU is also studied. Relevant procedures for effective sensing achieved with high energy efficiency are as follows. 
Step 1: Divide the whole sensor network into clusters (cluster formation) and update the clusters if any CR moves (cluster updating).

Step 2: Divide each cluster into disjoint subsets (subset formation).

Step 3: Minimize sensing energy by taking variable sensing time into consideration, because a sensor node receiving a signal with high signal-to-noise ratio (SNR) consumes less energy and takes less time in sensing, which means it goes to sleep for a longer time to save energy.

Step 4: Schedule the subsets by activating only one of the subsets while switching the others to sleep mode. The PU's historical activity data are used to determine the sleep time of the subsets. These procedures lead to energy-efficient sensor network operation for the CRN, which is shown in comparison with the SENDORA network and a CRN with the LEACH-C protocol.

Main focus of this paper are as follows.

1. We study an energy-efficient cluster updating and subset formation (CUSF) process for the operation of ad hoc CRs assisted by an infrastructure sensor network. The CRs randomly move in time and the subsets of the clusters in the sensor network are updated accordingly.

2. Only one subset in a cluster is active at a time, while the others switch to sleep mode. For further reduction of energy consumption, the actual sensor nodes for spectrum sensing are selected from the given active subset according to a separately proposed algorithm. Energy savings during spectrum sensing is a critical matter with a CRN including many sensor nodes. Most of the published works consider only communication energy or processing energy when evaluating the energy consumption of the network, so energy consumed during the sensing stage is often ignored. Though energy for each sensing is considerably less than communication energy, the short interval in the periodic sensing process of the CRNs makes it significantly important. Thus, minimization of sensing energy helps to prolong the lifetime of the sensor network.

3. Even the one active subset can be switched to sleep mode for a certain number of time slots by the proposed scheduling algorithm, based on the history of PU activity. This scheduling achieves additional energy efficiency at the cost of a slightly increased error in PU detection.

4. We study comprehensive energy consumption of the sensor network with the CUSF architecture. Overall energy consumption of the sensor network involves energy consumed in setup, sensing, sending, and sleep stages. In the literature, the energy consumed in network setup has mostly been ignored. However, due to free and frequent moves of the CRs and the subsequent CUSF process for each move, energy consumed in the setup stage is additionally considered.

\section{RELATED WORK}

Liu and Weiss proposed application of a sensor network for CR. However, they did not describe the architecture and the topology of the sensor network. Mercier proposed sensorassisted CR, namely a sensor network for dynamic and cognitive radio access (SENDORA), where information on PU activity detected by a separate sensor network is transmitted via a single sink to the CRN in multi-hops. The SENDORA network addressed a comprehensive perspective of a sensor-assisted CRN.

Nevertheless, it is subject to failure when the sink node breaks down, and it suffers from high energy consumption as well as high end-to-end delay because of multi-hop transmissions to the CR. Akan and Joshi, described a CR sensor network (CRSN) where the conventional wireless sensor nodes are equipped with $\mathrm{CR}$ functionality. The CRSN requires highly complicated sensor nodes, so the high cost of a CRSN makes it impractical.

A sensor network of clusters with a hierarchical routing protocol to increase network lifetime was reported by Huang. They showed, with many sensor nodes, reduction of energy consumption by hierarchical routing instead of flat routing. However, their work is not related to mobile (ad hoc) CRs. Heinzelman proposed an energy-efficient routing protocol with low end-to-end delay, e.g., low energy adaptive clustering hierarchy (LEACH). The LEACH protocol did not, however, consider the energy state of cluster heads and sensor nodes. To enhance energy efficiency of a sensor network, various approaches have appeared.

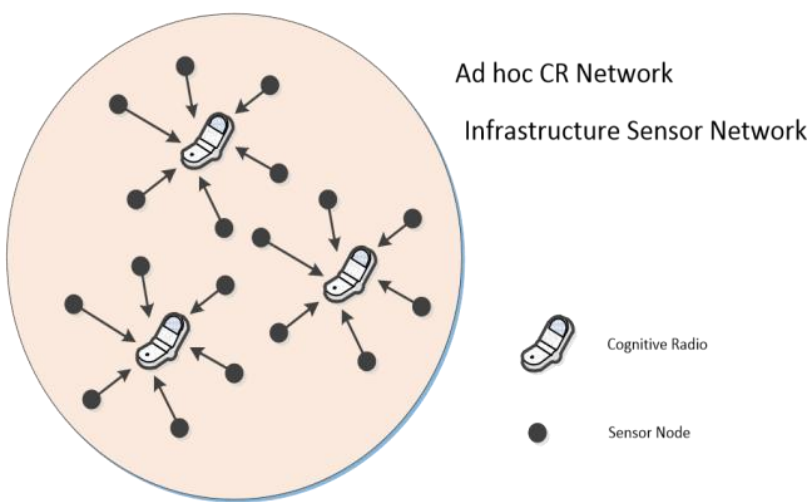

Fig. 1. Sensor-assisted CRN where CRs are surrounded by sensor nodes.

\section{SYSTEM DESCRIPTION}

Consider the sensor-assisted CRN with ad hoc CRs in Fig.1. Each mobile station acts as a CR that is surrounded by sensor nodes. It is assumed that the PU operates in a timeslotted fashion. A sensor node in active mode goes through quadruple $S$-stages (setup, sense, send, and sleep) in a time slot. The sensor nodes send (report) the sensing results directly to the CR that serves as a cluster head. Since an infrastructure sensor network is considered, positions of the sensor nodes are assumed to be known to the CR. It is also assumed that each sensor node knows its own position. Each 
CR has the ability to find its geolocation from an embedded GPS module. A CR may move in any direction within a predefined area. An error-free common control channel is assumed for the exchange of control information between nodes and the CR. All the CRs have the same communication range, and the sensor nodes have a different communication range, where $\mathrm{CR}$ communication range is greater than the sensor node communication range.

A CR broadcasts an advertisement (ADV) message which contains the identification number (ID) of the CR, its position, the nodes registered to the CR (Nodes), and a header field. The purpose of the header field is to differentiate the ADV message from other types of message or data.

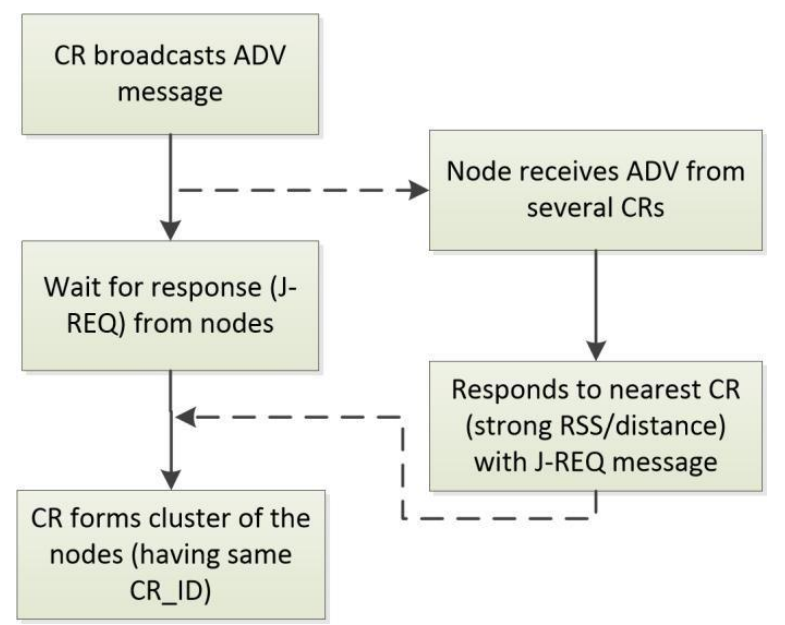

Fig. 3. Flow chart for cluster formation.

A node may receive multiple ADV messages from different CRs. In this case, the node will join the CR that is closest to it in order to consume the minimum transmission energy.

Note that a node knows the position(s) of the CR(s) via the $\mathrm{ADV}$ messages. If a node is equi-distant from two or more $\mathrm{CRs}$, it will join the $\mathrm{CR}$ with the smallest number of registered nodes to minimize waiting time for sending the sensing result. On receiving the joining request from the sensor node(s), the CR adds the node(s) to the list of registered nodes.

Now assuming the CR does not leave its position for one time slot. The cluster updating process takes place only when there is a change in $\mathrm{CR}$ position. The unclustered nodes join the nearest CR. A cluster can be decomposed into disjoint subsets, activating once subset of the nodes instead of all subsets in the cluster there by significantly reducing energy consumption by deactivating subsets to sleep modes. A subset of a cluster defined for this work is a group of nodes that covers the area of a cluster with minimum overlap.

After subset formation, the CR selects the subset that has the maximum total energy, and creates a time division multiple access (TDMA) schedule for the nodes in the selected subset. This scheduling information is transmitted to the sensor nodes in the form of beacon messages by the CR. Only the nodes included in a subset are active; all other nodes in other subsets remain in sleep mode. The order of time slots in a TDMA frame is assigned according to descending order of SNR values of the sensor nodes. Thus, the first time slot is assigned to the sensor node with the highest SNR, and so on.

ENERGY CONSUMPTION DETAIL: The energy consumed in the setup stage consists of the Setup, Sensing and Reporting stages. The processing energy at the senor nodes is ignored, because it is significantly smaller. The energy is consumed in advertising and sending joining requests to the $\mathrm{CR}$.

PU ACTIVITY: The time slots when the PU can be predicted to be in sleep mode including the active subsets. This prediction can be modeled by a two-state Markov Chain where the presence and absence of the PU are modeled by busy and idle states respectively. Based on the past sensing a history of the PU is created in the CR. As per this created history modes are predicted, this is combined with the individual sensing results of the sensor nodes. These patterns are recorded over a period of time based on which the prediction can be made.

THE END-TO-END DELAY: The end-to-end delay is defined as the time taken from sensing by the sensor nodes to the end of reporting received at the registered $\mathrm{CR}$. The LEACH-C protocol uses a centralized clustering approach for the selection of cluster heads. The cluster formation (network setup), sensing, and reporting of the LEACH-C protocol are similar to those of the CUSF process. By the LEACH-C protocol, sensing is performed at the sensor nodes, and then the results are reported to the cluster head (the $\mathrm{CR}$ in the $\mathrm{CRN}$ ) that aggregates the data for final forwarding to the base station. The end-to-end delay of the LEACH-C protocol comprises: i) the delay due to the formation of cluster heads by the central base station and subsequent dissemination of this information to the cluster nodes, ii) the delay at the sensor nodes caused by sensing and reporting, and iii) the delay at cluster heads from processing and transmission. The end-to-end delay of the SENDORA network is the combination of delays at the sensor nodes, the cluster heads, and the sink node. It is studied that the CRN with the CUSF process causes significantly lower delay, compared to the other protocols. The main reasons for the lower delay with the CUSF process are (i) smaller size of the subset resulting in lower transmission delay and (ii) the CR directly receiving sensing results from the sensor nodes.

The CRN with the CUSF process consumes the least amount of energy. That is due to having the least number of sensor nodes in sensing and sending. With the SENDORA network and the CRN adopting the LEACH-C protocol, additional energy consumption at the cluster head in aggregating data and transmitting them to the sink node for the secondary network is needed. However, the energy consumed for the additional process is comparatively negligible, so it is 
disregarded for comparison of energy consumption. The energy consumption of the network increases in each round because of the number of relocations of the CRs, accompanied by cluster updating and subset formation. With lesser rounds, the CRN with the CUSF process consumes lesser energy than the SENDORA network and LEACH-C Protocol.

\section{CONCLUSION}

In this paper, an ad hoc CRN with an energy-efficient process, namely the CUSF process, is studied. Via the CUSF process, clustering and further subset formation of the sensor nodes are performed. Multiple subsets are created in a cluster and only one subset is active in sensing to reduce energy consumption. For further reduction of energy consumption, the actual sensor nodes for spectrum sensing are selected in the given active subset. In addition, all the subsets, including the one active subset, switch to sleep mode for the duration of PU activity to achieve another reduction in energy consumption. A novel subset scheduling approach to achieve this goal is discussed. As a result, the CRN with the CUSF architecture consumes significantly less energy and incurs lower end-to-end delay in comparison with the SENDORA network and the CRN with the LEACH-C protocol.

\section{REFERENCES}

[1] F. C. Commission, "Facilitating opportunities for flexible, efficient, and reliable spectrum use employing cognitive radio technologies," Et docket, pp. 05-57, 2003.

[2] E. Hong, K. Kim, and D. Har, "Spectrum sensing by parallel pairs of cross-correlators and comb filters for OFDM systems with pilot tones," Sensors Journal, IEEE, vol. 12, pp. 2380-2383, 2012.

[3] S. Haykin, "Cognitive radio: brain-empowered wireless communications," Selected Areas in Communications, IEEE Journal on, vol. 23, pp. 201220, 2005.

[4] A. Ghasemi and E. S. Sousa, "Collaborative spectrum sensing for opportunistic access in fading environments," in New Frontiers in Dynamic Spectrum Access Networks, 2005. DySPAN 2005. 2005 First IEEE International Symposium on, 2005, pp. 131-136.

[5] X. Liu, B. G. Evans, and K. Moessner, "EnergyEfficient Sensor Scheduling Algorithm in Cognitive Radio Networks Employing Heterogeneous Sensors," Vehicular Technology, IEEE

[6] O. B. Akan, O. B. Karli, and O. Ergul, "Cognitive radio sensor networks," Network, IEEE, vol. 23, pp. 34-40, 2009.

[7] G. P. Joshi, S. Y. Nam, and S. W. Kim, "Cognitive radio wireless sensor networks: applications, challenges and research trends," Sensors, vol. 13, pp. 11196-11228, 2013.

[8] R. Deng, J. Chen, C. Yuen, P. Cheng, and Y. Sun, "Energy-efficient cooperative spectrum sensing by optimal scheduling in sensor-aided cognitive radio networks," Vehicular Technology, IEEE

[9] J. Kim, X. Lin, N. B. Shroff, and P. Sinha, "Minimizing delay and maximizing lifetime for wireless sensor networks with anycast," IEEE/ACM Transactions on Networking (TON), vol. 18, pp. 515528, 2010.

[10] G. Anastasi, M. Conti, and M. Di Francesco, "Extending the lifetime of wireless sensor networks through adaptive sleep," Industrial Informatics, IEEE Transactions on, vol. 5, pp. 351-365, 2009.

[11] O. Younis and S. Fahmy, "HEED: a hybrid, energyefficient, distributed clustering approach for ad hoc sensor networks," Mobile Computing, IEEE Transactions on, vol. 3, pp. 366-379, 2004.

[12] J. Chen, J. Li, S. He, Y. Sun, and H.-H. Chen, "Energy-efficient coverage based on probabilistic sensing model in wireless sensor networks," Communications Letters, IEEE, vol. 14, pp. 833-835, 2010.

[13] E. C. Y. Peh, L. Ying-Chang, G. Yong Liang, and P. Yiyang, "Energy-Efficient Cooperative Spectrum Sensing in Cognitive Radio Networks," in Global Telecommunications Conference (GLOBECOM 2011), 2011 IEEE, 2011, pp. 1-5.

[14] H. N. Pham, Y. Zhang, P. E. Engelstad, T. Skeie, and F. Eliassen, "Energy minimization approach for optimal cooperative spectrum sensing in sensor-aided cognitive radio networks," in Wireless Internet Conference (WICON), 2010 The 5th Annual ICST, 2010, pp. 1-9.

[15] X. Li, D. Wang, X. Mao, and J. McNair, "On the accuracy of maximum likelihood estimation for primary user behavior in cognitive radio networks," Communications Letters, IEEE, vol. 17, pp. 888-891, 2013.

[16] T. Camp, J. Boleng, and V. Davies, "A survey of mobility models for ad hoc network research," Wireless communications and mobile computing, vol. 2, pp. 483-502, 2002.

[17] Muhammad Usman, Dongsoo Har, Senior Member, IEEE, and Insoo Koo, Member, IEEE, "EnergyEfficient Infrastructure Sensor Network for Ad Hoc Cognitive Radio Network “ 